\title{
Mask roughness induced LER: geometric model at long correlation lengths
}

\author{
Brittany M. McClinton ${ }^{1}$ and Patrick P. Naulleau ${ }^{2}$ \\ ${ }^{1}$ Dept. of Electrical Engineering, University of California, Berkeley, CA 94720 \\ ${ }^{2}$ Center for X-Ray Optics, Lawrence Berkeley National Laboratory, Berkeley, CA 94720
}

\begin{abstract}
Collective understanding of how both the resist and line-edge roughness (LER) on the mask affect the final printed LER has made significant advances. What is poorly understood, however, is the extent to which mask surface roughness couples to image plane LER as a function of illumination conditions, NA, and defocus. Recently, progress has been made in formulating a simplified solution for mask roughness induced LER. Here, we investigate the LER behavior at long correlation lengths of surface roughness on the mask. We find that for correlation lengths greater than $3 \lambda / \mathrm{NA}$ in wafer dimensions and CDs greater than approximately $0.75 \lambda / \mathrm{NA}$, the previously described simplified model, which remains based on physical optics, converges to a "geometric regime" which is based on ray optics and is independent of partial coherence. In this "geometric regime", the LER is proportional to the mask slope error as it propagates through focus, and provides a faster alternative to calculating LER in contrast to either full 2D aerial image simulation modeling or the newly proposed physical optics model. Data is presented for both an NA $=0.32$ and an NA $=0.5$ imaging system for CDs of 22-nm and 50-nm horizontal-line-dense structures.
\end{abstract}

Keywords: extreme ultraviolet, lithography, line-edge roughness, mask roughness, speckle

\section{INTRODUCTION}

It is becoming increasingly challenging to meet the requirements for line-edge roughness (LER) as extremeultraviolet lithography (EUVL) pushes to ever smaller critical dimensions (CDs). For the 22-nm half-pitch node, the International Roadmap for Semiconductors [1] calls for less than $1.2 \mathrm{~nm}$ LER. Current state-of-the-art EUVL tools can consistently achieve only about $3 \mathrm{~nm}$ LER. Critical to reaching the industry's goal is a more complete understanding of the principle causes of LER.

Although LER has traditionally been viewed as a resist-limited effect, as CDs shrink mask contributors become more and more important. For EUVL in particular, three major contributors (resist LER, mask LER, and mask roughness induced LER) must be taken into account. Analytic tools have been developed to predict both resist LER and mask LER $[2,3]$. While significant work has been made in understanding how mask roughness couples to speckle in the aerial image and hence to LER [4-9], the extent to which it currently factors into LER limits remains incompletely understood. Recently, progress has been made in formulating a simplified solution for mask roughness induced LER [10]. Given numerical aperture (NA), illumination conditions, defocus and mask roughness characteristics (well defined by replicated surface roughness (RSR) and correlation length), the clear-field speckle statistics calculated from a one-time 2D aerial image simulation can be quickly coupled to any feature size through fast $1 \mathrm{D}$ aerial image simulations of the image-log-slope (ILS) and relative intensity to predict the mask roughness induced LER. This method is a significant improvement in speed and ease over the previous method where one needed to conduct full 2D aerial image simulations in one software package and then extract the LER in another. Here, we propose a further simplification to the prediction of mask roughness induced LER in the limit of long correlation lengths of surface roughness on the mask. We show that in this limit for correlation lengths greater than $3 \lambda / \mathrm{NA}$ in wafer dimensions and CDs greater than approximately $0.75 \lambda / \mathrm{NA}$, the simplified model which remains based on wave optics converges to a "geometric regime" which is based on ray optics and is independent of partial coherence. In this "geometric regime", the LER is proportional to the mask slope error as it propagates through focus, and provides a yet faster alternative to predicting LER. 


\section{MASK ROUGHNESS INDUCED LER - A GEOMETRIC LIMIT}

As discussed elsewhere $[4,7,11]$ and only briefly presented here, surface roughness on a reflective mask geometrically induces phase roughness on the wavefront that due to the reflection is a factor of two larger than the actual surface deviations. For EUVL, this is detrimental since the operating wavelength is nominally $13.5 \mathrm{~nm}$ and very small roughness on the multi-layer mask can significantly modulate the phase. When reimaging the mask to the wafer in a lithographic process that is fundamentally band-limited, the resulting image is an imperfect replication of the mask object that is sensitive to the phase errors on it. Each point on a rough mask acts as a scattering point inducing a phase delay with respect to its neighbor so that taken across the image plane, there are many resulting overlapping amplitude spread functions that differ in phase from one to another and cause a complex interference pattern, the strength of which is modulated by the coherence factor. These interference events manifest themselves as intensity variations, or speckle, at scales down to the resolution limit of the objective [12]. By specifying the objective NA, coherence factor $\sigma$, defocus, and, of course, mask roughness, the statistical nature of the speckle is well described.

The macroscopic properties of mask roughness are well determined when the replicated surface roughness (RSR) and the correlation length are known. Here, we refer to the RSR as the standard deviation of the surface height across the mask, where we assume the top surface height deviations are replicated through the various layers comprising the multilayer. This geometric height difference from a perfectly flat mask imparts a phase delay, giving rise to a phase roughness along the wavefront. The correlation length, in contrast, is the degree to which the surface roughness is self-similar laterally on the mask. It is a measure of the extent to which the roughness is dephased laterally across the wavefront with respect to itself, and it governs the spatial frequency content of the roughness on the mask. Thus, for example, greater height variations (RSR) and lesser self-similarity across the mask (correlation length) mean that the mask is "rougher" in a statistical sense. These mask roughness characteristics, in combination with the parameters of the objective NA, coherence factor $\sigma$, and defocus, well-determine the speckle statistics, which we express as $S$ (see Fig. 1).

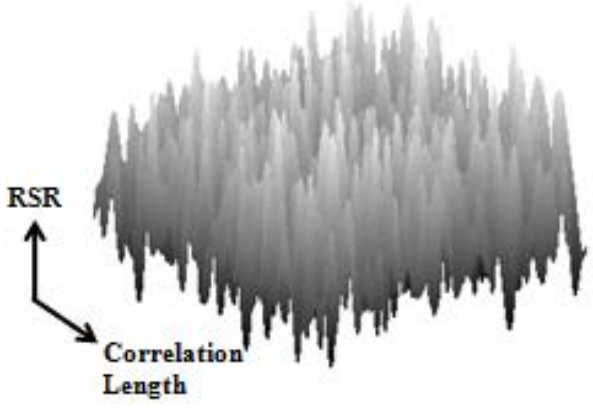

Mask Roughness

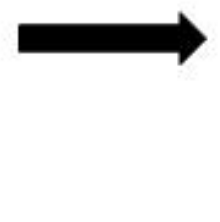

Speckle in Aerial Image

Fig. 1. Mask roughness statistics combined with illumination and band-limited imaging well determines speckle statistics in the aerial image.

Recently, it has been proposed that the LER can be expressed by a simplified solution [10]. The simplified model offers a distinct advantage over the traditional method of calculating mask roughness induced LER by brute force, which involves conducting full 2D aerial image simulations in commercially available software (such as PROLITH [13] or Panoramic [14]), followed by extracting the LER from the computed aerial image through offline analysis using a software package such as SuMMIT [15]. The simplified model, on the other hand, requires only a one-time 2D aerial image simulation through focus of the clear-field rough mask to get the mask roughness-specific clear-field speckle. This speckle then can be coupled to LER by using fast 1D aerial image simulations (e.g., using PROLITH) to get the feature- and illumination-specific ILS as it varies through focus. From here, the full parameter space can be reached by analytic extension. 


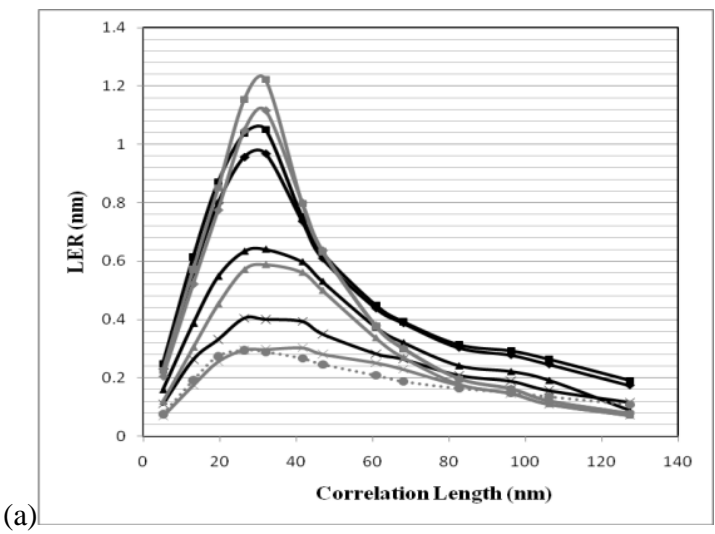

(a)

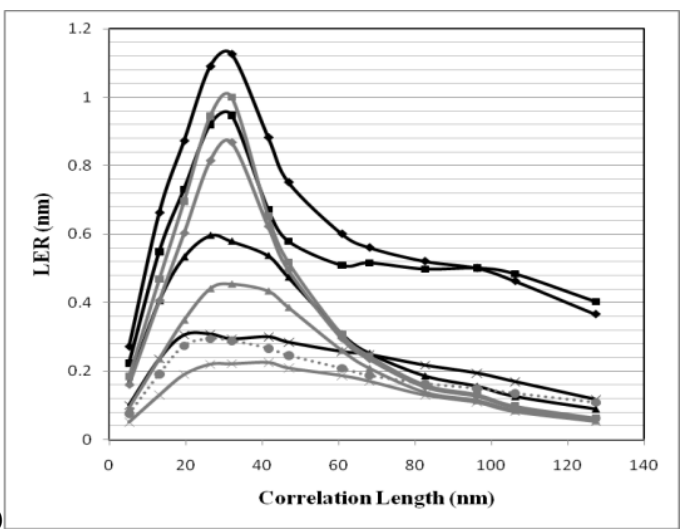

(b)
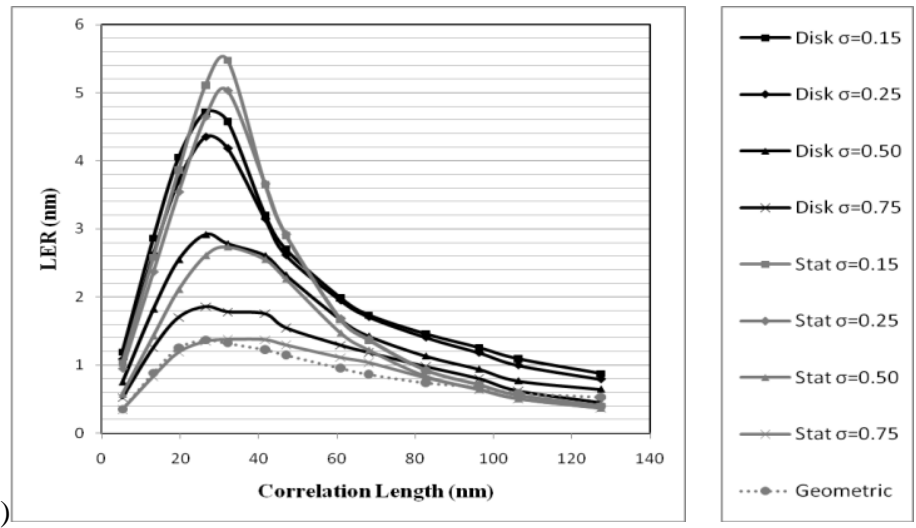

(d)

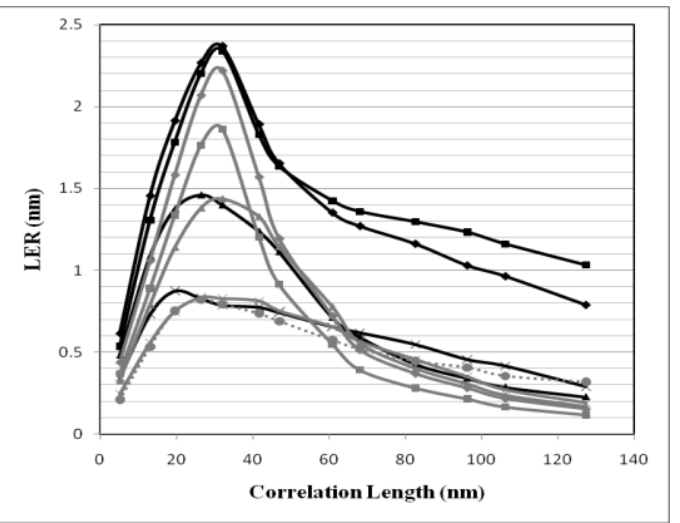

Fig. 2. Simplified LER ("Stat" indicated in gray) for the calculation of LER based on Eq. 2 in comparison with Simulated LER ("Disk" indicated in black) for RSR $50 \mathrm{pm}$, a variety of illuminations, a variety of correlation lengths, for (a) $50 \mathrm{~nm}$ hld for RSR 50 pm, (b) $50 \mathrm{~nm}$ hld for RSR $230 \mathrm{pm}$, (c) $22 \mathrm{~nm}$ hld for RSR $50 \mathrm{pm}$, (d) $22 \mathrm{~nm}$ hld for RSR $230 \mathrm{pm}$. All were at a defocus setting of $50 \mathrm{~nm}$, except for (d) which was at $-30 \mathrm{~nm}$ defocus.

The results from our earlier study [10] are reproduced in Fig. 2 for reference, comparing the fully simulated LER and the LER calculated from the simplified model. This was for an optical system with NA $=0.32$, four different illumination conditions $(\sigma=0.15,0.25,0.50,0.75)$, two RSR settings of $230 \mathrm{pm}$ and $50 \mathrm{pm}$, for $22 \mathrm{~nm}$ and $50 \mathrm{~nm}$ lines and spaces, at a defocus of $-50 \mathrm{~nm}$, through a variety of correlation lengths $(5,13,20,26,32,42,47,61,68,83,96,106,127 \mathrm{~nm})$, and a roughness exponent of infinity. We note that the effect of the roughness exponent on the simplified model has also been explored and found not to effect the quality of the comparison [10]. The $22 \mathrm{~nm}$ case with an RSR of $230 \mathrm{pm}$ had to be evaluated at a defocus of $-30 \mathrm{~nm}$, due to corrupted data further out of focus. For each illumination setting, the data were well-behaved through focus, exhibiting no crossover from one correlation length to another. Therefore the data at one defocus setting is consistent with how the LER behaves at other defocus settings as well. In all cases, we notice a strong peak in the LER for mid-spatial frequency roughnesses ( $26 \mathrm{~nm}$, wafer dimensions), about the resolution of the

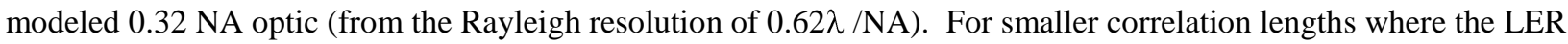
decreases, the objective lens provides a filtering benefit in that the high spatial frequencies of the scattering halo surrounding the diffracted orders fall out of the pupil. The LER again decreases, but much more gradually, on the other side of the peak. Here, all the light is captured by the pupil; this adverse effect, however, is mitigated due to a decreasing rate of change of the induced phase roughness, and, for correlation lengths greater than $3 \lambda / \mathrm{NA}$ in wafer dimensions, and CDs greater than approximately $0.75 \lambda / \mathrm{NA}$, becomes roughly independent of partial coherence. In this limit, a geometric model based on ray optics should be valid since interference effects no longer play an important role. In this "geometric regime", we assume the LER to be proportional to the mask slope error as it propagates through focus (see Fig. 3). 


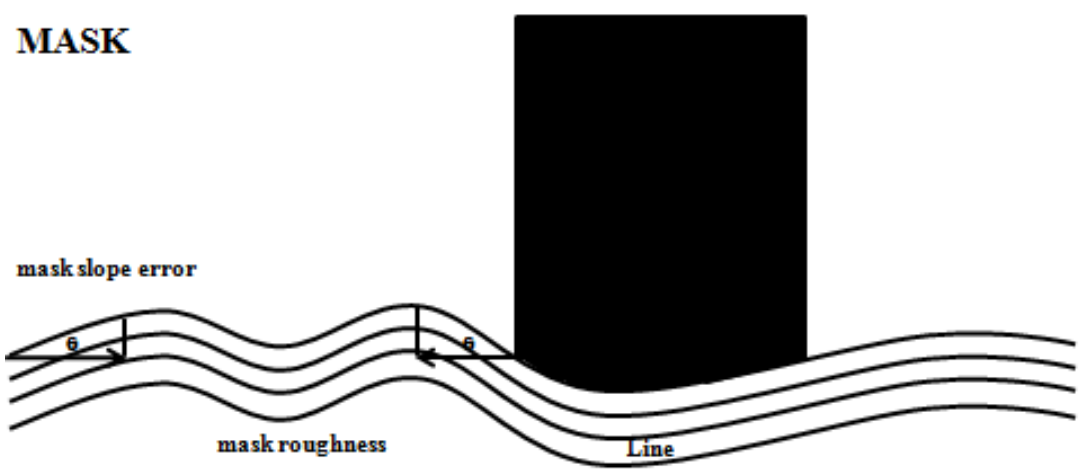

Fig. 3. In the "geometric regime", the LER is proportional to the mask slope error as it propagates through focus.

\section{MODELING APPROACH}

In this section we explore the validity of the geometric model by directly comparing it to LER values extracted from full $2 \mathrm{D}$ aerial image simulations. In the comparison, we consider an aberration-free optical system with $\mathrm{NA}=0.32$. For the mask we consider RSR values of 50 and $230 \mathrm{pm}$ and correlation lengths ranging from 5 to $127 \mathrm{~nm}$, and roughness exponent of infinity. To build up a significant statistical ensemble, 10 random realizations of the mask for each RSR / correlation length pair were considered. Assuming an illumination wavelength of $13.5 \mathrm{~nm}$, each topographic height map surface was converted to a phase perturbation. The mask was modeled with $1024 \mathrm{x} 1024$ pixels at $1 \mathrm{~nm} /$ pixel assuming $1: 1$ imaging to the wafer plane.

For the traditional method of computing LER, which is calculated directly from the 2D aerial-image simulations, each clear-field rough mask realization described above was overlaid (multiplied) with an ideal binary amplitude grating, for both $22 \mathrm{~nm}$ and $50 \mathrm{~nm}$ line-space patterns. Simulations were carried out through focus for 4 different disk illuminations: coherence factors $\sigma=0.15,0.25,0.50$, and 0.75. The LER was then extracted in SuMMIT [15], using only nested features for LER analysis, avoiding the outer $200 \mathrm{~nm}$ border, using the proper threshold for each sigma value to provide proper sizing of the lines at best focus.

In considering the geometric model, we assume that the LER is proportional to the mask slope error as it propagates through focus. We therefore first constructed the point-spread-function of an aberration-free optical system the same as that already investigated above [10], with an objective $\mathrm{NA}=0.32$ and assuming an illumination wavelength of $13.5 \mathrm{~nm}$. We then convolved this with the topographical height map of the clear-field rough mask to get at how the mask is seen from the image plane (see Fig. 4). This height map is dependent only on the mask roughness properties, and was repeated on the same statistical ensemble of rough masks used to produce the data represented in Fig. 2; that is, masks for both an RSR of $50 \mathrm{pm}$ and $230 \mathrm{pm}$, and a variety of correlation lengths $(5,13,20,26,32,42,47,61,68,83,96,106$, $127 \mathrm{~nm}$ ), a roughness exponent of infinity, with 10 random realizations of the mask for each RSR / correlation length pair. After the convolution performed on each mask, we then took the slope and propagated through focus to yield the geometric prediction which is also plotted in Fig. 2. 


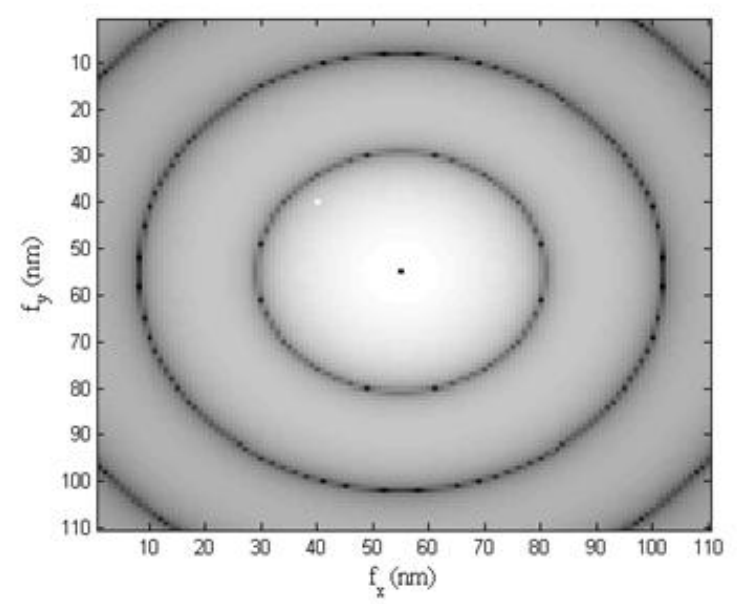

PSF

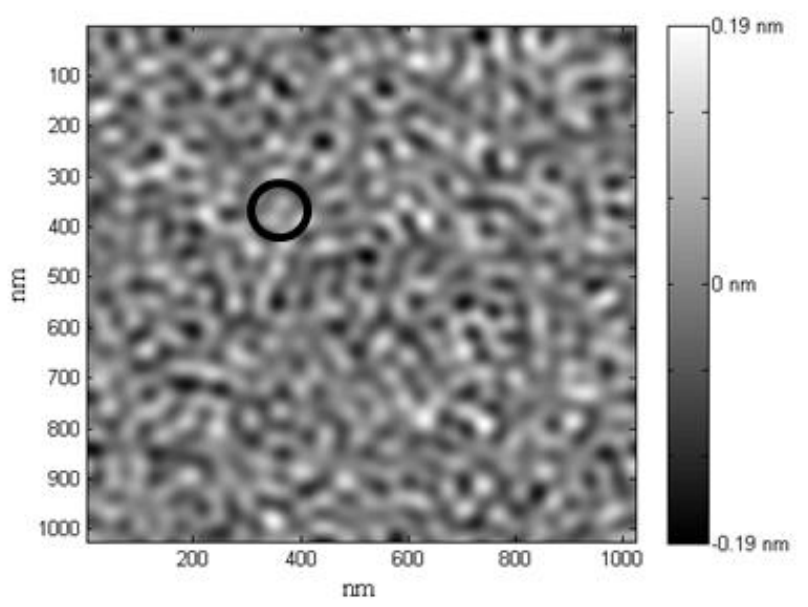

Rough Mask

Fig. 4. To generate the "geometric" model, we constructed the point-spread-function of our imaging system (shown in log-scale on the left) and convolved it with the topographical height map of the clear-field rough mask to get how the mask is seen from the wafer plane (shown on the right, along with a circle representing the first null of the Airy pattern in the PSF, radius of 26nm). We then took the slope across the mask to get the mask slope error, and propagated through focus appropriately.

\section{VERIFICATION OF THE GEOMETRIC MODEL}

It is evident that the geometric model provides a good prediction of the LER for longer correlation lengths.

We note that even in the case where the $\mathrm{CD}$ is less than $0.75 \lambda / \mathrm{NA}$, the geometric model holds well with those illumination conditions which are less coherent $(\sigma=0.50$, and 0.75 in Fig. 2$)$.

To further test this model, we also consider an NA of 0.5 . We repeated all the data for all three methods (fully simulated LER, LER from the simplified model, and LER for the geometric model) for masks of an RSR 50 pm with the same set of correlation lengths, and roughness exponent of infinity for the same illumination conditions $(\sigma=0.15,0.25,0.50$, and 0.75), for both $22 \mathrm{~nm}$ and $50 \mathrm{~nm}$ lines and spaces. This data is seen in Fig. 5 (a) for a defocus of $-50 \mathrm{~nm}$ for $50 \mathrm{~nm}$ lines and spaces, and (b) for a defocus of $-30 \mathrm{~nm}$ on $22 \mathrm{~nm}$ lines and spaces. Again, the geometric model provides a good prediction of the LER for roughness correlation lengths greater than $3 \lambda / \mathrm{NA}$ in wafer dimensions. In this case since both CDs considered $(22 \mathrm{~nm}$ and $50 \mathrm{~nm}$ ) are now greater than approximately $0.75 \lambda / \mathrm{NA}$, all illumination conditions converge well to the geometric model for both CDs.

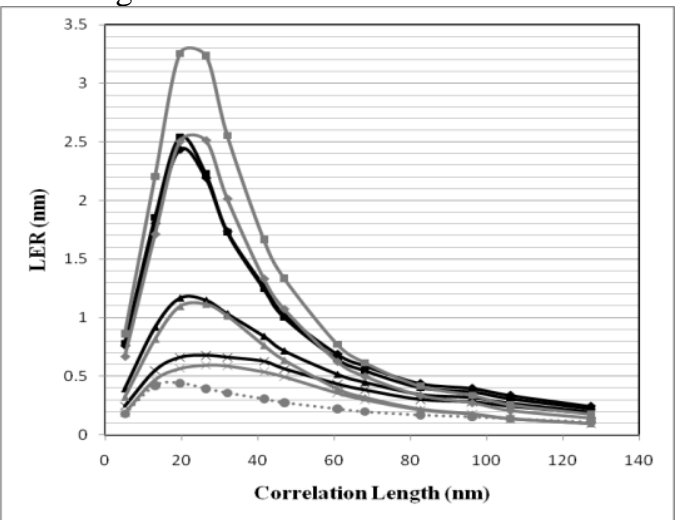

(a)

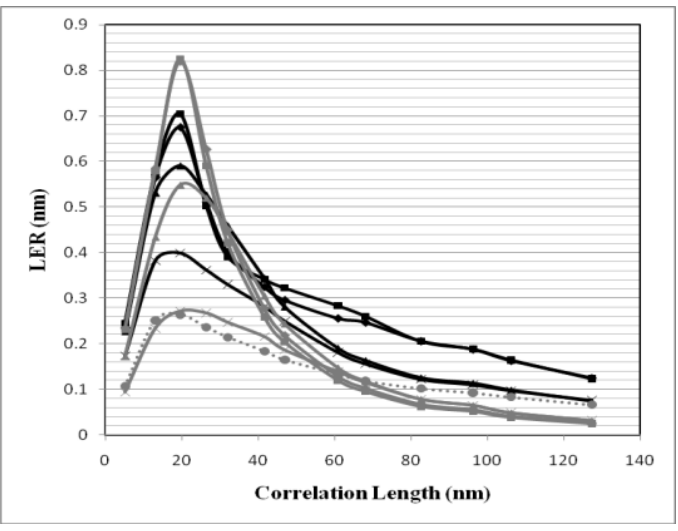

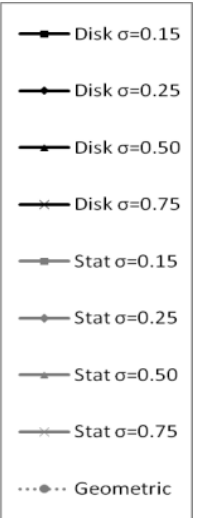

Fig. 5. Simulated LER vs. Simplified LER for an NA = 0.5 system, RSR $50 \mathrm{pm}$, a variety of illuminations, through correlation length, (a) at a defocus of $-50 \mathrm{~nm}$ for $50 \mathrm{~nm}$ lines and spaces, and (b) at a defocus of $-30 \mathrm{~nm}$ for $22 \mathrm{~nm}$ lines and spaces. 


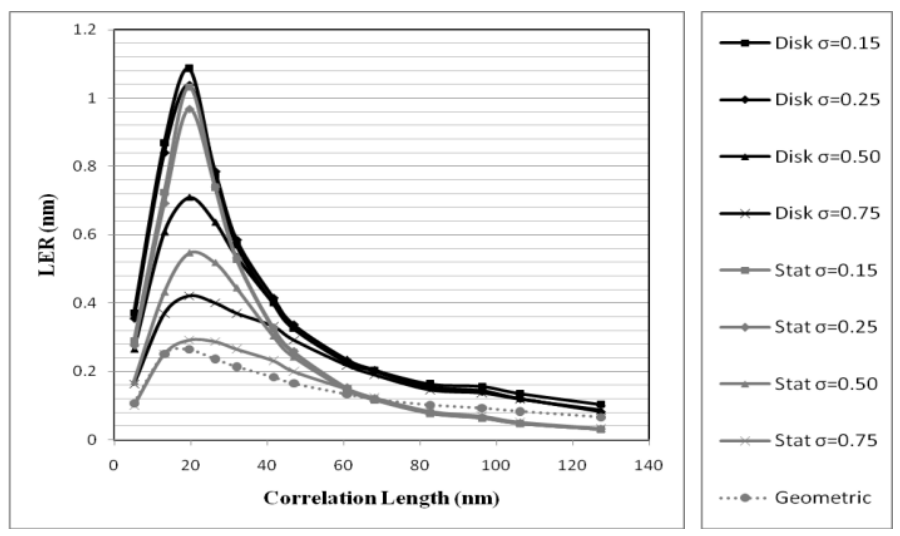

Fig. 6. Simulated LER vs. Simplified LER for an $\mathrm{NA}=0.5$ system, RSR $50 \mathrm{pm}$, a variety of illuminations, through correlation length, at a defocus of $-30 \mathrm{~nm}$ for $50 \mathrm{~nm}$ lines and spaces.

\section{CONCLUSIONS}

We have shown that for roughness on the mask with correlation lengths greater than $3 \lambda / \mathrm{NA}$ in wafer dimensions and CDs greater than approximately $0.75 \lambda / \mathrm{NA}$, the physical optics-based simplified model converges to a geometric, ray optics regime which is independent of partial coherence. In comparison to either the fully simulated LER or even the LER calculated from the simplified model, the geometric model provides an advantage in the ease and speed of its calculation. We note, however, that our implementation of the geometric model still involves a convolution with the physical optics point spread function in order to determine the relevant image plane wavefront slopes.

We also notice that for the NA $=0.5$ imaging system, the location of the peak in LER has shifted to the slightly lower mid-spatial frequency of correlation length of $20 \mathrm{~nm}$. This is consistent with the earlier prediction [10] that the location of this peak is independent of the feature size, and is tied directly to the resolution of the objective (about $0.62 \lambda / \mathrm{NA}$ ). Therefore, masks with surface roughness of $20 \mathrm{~nm}$ correlation length should be avoided. In order to meet the ITRS [1] benchmark of $1.2 \mathrm{~nm}$ total combined LER, and assuming an RSR of $50 \mathrm{pm}$, the correlation length should be greater than approximately $50 \mathrm{~nm}$ to limit the mask roughness induced LER contribution to under $0.3 \mathrm{~nm}$. Surprisingly, the stringency of this correlation length requirement is relaxed for the $\mathrm{NA}=0.5$ system in comparison to the $\mathrm{NA}=0.32$ system, where the LER peak was much broader and shifted slightly towards lower spatial frequencies. This allows shorter correlation lengths to be much more tolerable in the NA $=0.5$ case. In addition, there is the added benefit of greatly reduced contribution of mask roughness induced LER for all correlation lengths for the NA $=0.5$ system. This is due to the fact that the higher NA does a better job replicating the pure phase function, thus reducing the coupling to speckle. This is in opposition to the effect on mask LER where the higher NA will also do a better job replicating the mask LER (less filtering of the mask LER).

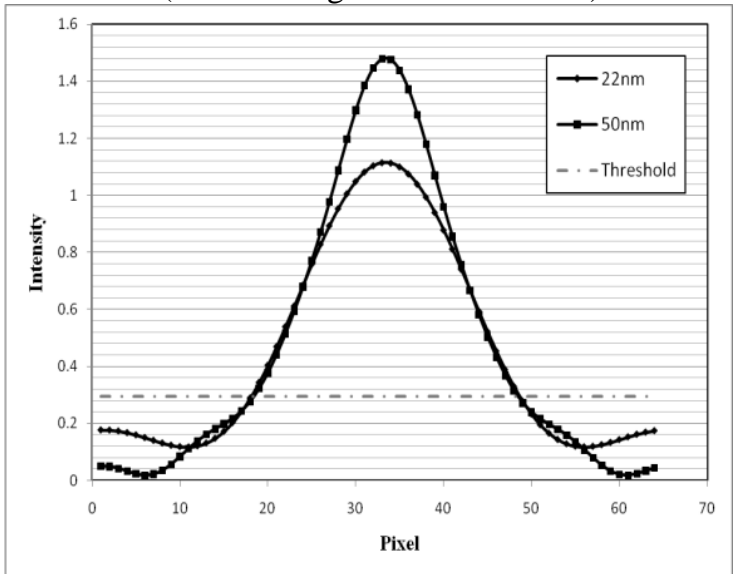

Fig. 7. 1D Aerial images for a partial coherence of $\sigma=0.15, \mathrm{NA}=0.5$, as a function of horizontal position for $50 \mathrm{~nm}$ lines and spaces at a defocus of $-50 \mathrm{~nm}$ where the horizontal scale is $1.56 \mathrm{~nm} /$ 1 pixel, and for $22 \mathrm{~nm}$ lines and spaces at a defocus of $-30 \mathrm{~nm}$ where the horizontal scale is $0.6875 \mathrm{~nm} / 1$ pixel.

We note that in Fig. 5 for the NA $=0.5$, the LER for $22 \mathrm{~nm}$ lines and spaces (at a defocus of $-30 \mathrm{~nm}$ ) seems much better than that for $50 \mathrm{~nm}$ (at a defocus of $-50 \mathrm{~nm}$ ), disproportionate to the slight shift in defocus between them. We therefore 
recalculated all the data for the case of $50 \mathrm{~nm}$ lines and spaces at a defocus setting of $-30 \mathrm{~nm}$ for direct comparison in Fig. 6. We see that despite the shift in defocus, the LER for $50 \mathrm{~nm}$ lines and spaces remains worse than for the $22 \mathrm{~nm}$ case. To investigate more closely, we directly plotted the 1D aerial image simulations in Fig. 7. The diamond marker is the aerial image of $22 \mathrm{~nm}$ at a defocus of $-30 \mathrm{~nm}$ and the square marker is that for $50 \mathrm{~nm}$ at a defocus of $-50 \mathrm{~nm}$ just for one disk illumination of $\sigma=0.15$. From this, it is clear that the $50 \mathrm{~nm}$ lines and spaces begin to exhibit a feature that is indicative of higher NA systems for larger $\mathrm{CD}$, where higher diffracted orders (above order \pm 1 ) begin entering the pupil and add characteristic sidelobes to the aerial image. Because these sidelobes are present in the $50 \mathrm{~nm}$ feature in the threshold region, the ILS is adversely affected, and manifests itself in an LER value which is inferior to the smaller 22 $\mathrm{nm}$ feature. Although this potential problem could be readily addressed by proper biasing at the mask, it is interesting to note that the ability of the simplified model to accurately predict this anomalous behavior is indicative of the generality of the method.

The authors are grateful for the support of this work by the National Science Foundation EUV Science and Technology Center. This research was also supported in part by SEMATECH and carried out at Lawrence Berkeley National Laboratory's Advanced Light Source. This work was supported by the Director, Office of Science, of the U.S. Department of Energy under Contract No. DE-AC02-05CH11231.

\section{REFERENCES}

1. International Technology Roadmap for Semiconductors, 2009 ed., http://public.itrs.net/.

2. G. Gallatin, "Resist Blur and Line Edge Roughness," Proc. SPIE 5754, 38-52 (2005).

3. P. Naulleau and G. Gallatin, "Line-edge roughness transfer function and its application to determining mask effects in EUV resist characterization,” Appl. Opt. 42, 3390-3397 (2003).

4. T. Milster and N. Beaudry, "Scattering and Coherence in EUVL," in Emerging Lithographic Technologies II, Y. Vladimirsky, ed., Proc. SPIE 3331, 537-543 (1998).

5. N. Beaudry and T. Milster, "Effects of mask roughness and condenser scattering in EUVL systems," in Emerging Lithographic Technologies III, Y. Vladimirsky, ed., Proc. SPIE 3676, 653-662 (1999).

6. N. Beaudry and T. Milster, "Effects of object roughness on partially coherent image formation," Opt. Lett. 25, 454456 (2000).

7. P. Naulleau, "Relevance of mask-roughness-induced printed line-edge roughness in recent and future extremeultraviolet lithography tests," Appl. Opt. 43, 4025-4032 (2004).

8. P. Naulleau and G. Gallatin, "Spatial scaling metrics of mask-induced line-edge roughness," J. Vac. Sci. Technol. B 26, 1903-1910 (2008).

9. P. Naulleau, D. Niakoula, and G. Zhang, "System-level line-edge roughness limits in extreme ultraviolet lithography,” J. Vac. Sci. Technol. B 26, 1289-1293 (2008).

10. B. McClinton and P. Naulleau, "Mask roughness induced LER: a rule-of-thumb," J. Micro/Nanolith. MEMS and MOEMS 9, 041208 (2010).

11. P. Naulleau and S. George, "Implications of image plane line-edge roughness requirements on extreme ultraviolet mask specifications," Proc. SPIE 7379, 1-11 (2009).

12. J. W. Goodman, Statistical Optics (Wiley, New York, 1985), Chap. 7, pp. 286-360. 
13. PROLITH is a registered trademark of KLA-Tencor Corporation, 160 Rio Robles, San Jose, Calif. 95134.

14. Panoramic Technology, Inc., "EM-suite: core lithography simulation package," (2009), see

http://www.panoramictech.com.

15. EUV Technology Corp., http://www.euvl.com/summit.

\section{DISCLAIMER}

This document was prepared as an account of work sponsored by the United States Government. While this document is believed to contain correct information, neither the United States Government nor any agency thereof, nor The Regents of the University of California, nor any of their employees, makes any warranty, express or implied, or assumes any legal responsibility for the accuracy, completeness, or usefulness of any information, apparatus, product, or process disclosed, or represents that its use would not infringe privately owned rights. Reference herein to any specific commercial product, process, or service by its trade name, trademark, manufacturer, or otherwise, does not necessarily constitute or imply its endorsement, recommendation, or favoring by the United States Government or any agency thereof, or The Regents of the University of California. The views and opinions of authors expressed herein do not necessarily state or reflect those of the United States Government or any agency thereof or The Regents of the University of California. 